\title{
Call for nominations for the Heinrich-Emanuel-Merck Award
}

In the promotion of analytical science, Merck KGaA, Darmstadt, Germany announces the HEINRICH-EMANUELMERCK AWARD FOR ANALYTICAL SCIENCE. The prize which in 2010 will be awarded for the 10th time since 1988, has achieved remarkable recognition not least by the impressive list of previous award winners, recently including Dr. A. Makarov, Germany; and Prof. S. Nie, USA (2007), and Prof. Y. Baba, Japan (2004).

The prize was donated to mark the centenary of the first standardization of analytical methods brought about by Merck chemist Dr. Carl Krauch in his book "The Testing of the Purity of Chemical Reagents". The award bears the name of Heinrich Emanuel Merck, who defined the company's commitment to quality by the claim "I shall always guarantee the purity of my preparations".

In 2010 the prize will be opened to analytical science in general. It is intended for chemists up to the age of 45 , working in particular on developing new analytical methods and their applications in areas of human interest. Their work should be directed towards the improvement of our conditions of life, providing solutions to analytical problems in the areas of life sciences, material sciences or environment.

The prize is endowed with 15000 Euro and will be awarded at the 3rd EuCheMS Chemistry Congress in Nürnberg, Germany, 29 August-2 September 2010.
Applications should be submitted in the form of one original paper, i.e. published own research results not older than 3 years, preferably in English. A brief scientific curriculum vitae including the applicant's age should accompany all applications. The applications will be evaluated by a jury of internationally recognized analytical scientists.

Applications should be submitted by 31 March 2010 to the Chairman of the Jury of the Heinrich-Emanuel-MerckAward

Prof. Dr. Reinhard Niessner

Technical University of Munich

Institute of Hydrochemistry

Marchioninistrasse 17

81377 Munich, Germany

Phone: +49-89-218078231

Fax: +49-89-218078255

E-Mail: reinhard.niessner@ch.tum.de Internet: http://www.hem-award.merck.de

(Source: http://www.hem-award.merck.de accessed 14 Dec 2009) 